\title{
Physiological and psychological responses to a university fitness session
}

\author{
S. Grant MSc, G. Armstrong BSc, R. Sutherland BSc, J. Wilson*, T. Aitchisont BSc, \\ E. Pault BSc and S. Henderson MSc \\ Department of Physical Education and Sports Science, *Institute of Physiology, University of Glasgow, \\ tDepartment of Statistics, Glasgow, UK
}

\begin{abstract}
The purpose of this study was to examine the physiological and psychological responses to a university fitness session entitled 'popmobility'. A popmobility session consists of $20 \mathrm{~min}$ of aerobic activities, $5 \mathrm{~min}$ of local muscular endurance exercises and 5 min of flexibility exercises. Ten regular participants of these sessions, women of mean(s.d.) age 21.2(1.5) years, took part in the study. A maximal oxygen uptake $\left(\dot{V}_{\mathrm{O}_{2} \text { max }}\right)$ treadmill test was performed by each subject to obtain $\dot{V}_{\mathrm{O}_{2} \text { max }}$ and maximum heart rate values. In a laboratory, heart rate and $\dot{V}_{\mathrm{O}_{2}}$ were measured throughout a popmobility session for each subject. Rate of perceived exertion (RPE) was measured every $5 \mathrm{~min}$ throughout the session. The mean intensity of the aerobic part of the session ranged from $67.7-82.6 \%$ of the subject's $\dot{V} \mathrm{O}_{2 \max }$ (mean of $76.4 \% \quad \dot{V}_{\mathrm{O}_{2} \text { max }}$ ). The mean heart rate reserve for the aerobic section was $75.6 \%$. While the relative oxygen consumption remained fairly static during the aerobic section, the RPE score rose. The mean(s.d.) total energy expenditure was $236.6(28.4) \mathrm{kcal}$ (range 203-288). The popmobility session is of adequate intensity to improve the aerobic fitness of its participants. Heart rate, as used as a measure of intensity during a popmobility session, would appear to be a fairly accurate indicator of intensity. However, the use of RPE for exercise prescription in popmobility sessions is inappropriate. Popmobility could also be useful in a weightreduction programme.
\end{abstract}

Keywords: Aerobic training, indoor fitness sessions, energy expenditure

The general population is being encouraged to exercise regularly to improve/maintain various components of health related fitness. Each week over 1000 people take part in the University of Glasgow fitness session entitled popmobility. In a popmobility session exercises are carried out in rhythm with music. A popmobility session consists of $20 \mathrm{~min}$ of aerobic activities, $5 \mathrm{~min}$ of local muscular endurance exercises and $5 \mathrm{~min}$ of flexibility exercises. Aerobic dance sessions are similar to popmobility sessions. Previous studies have shown that aerobic dance

Address for correspondence: Mr S. Grant, Department of Physical Education and Sports Science, Stevenson Building, 77 Oakfield Avenue, University of Glasgow, Glasgow G12 8LT, UK

(C) 1993 Butterworth-Heinemann Ltd 0306-3674/93/030162-05 sessions can stress the cardiorespiratory system sufficiently to produce training effects ${ }^{1-3}$. These effects have been reflected in alterations in maximal oxygen uptake $\left(\dot{V}_{\mathrm{O}_{2} \max }\right)$, ranging from nonsignificant increases ${ }^{4}$ to highly significant increases of $23 \%{ }^{3}$.

According to the American College of Sports Medicine (ACSM) ${ }^{5}$, aerobic fitness can be improved by exercising large muscle groups continuously for $20-60$ min duration, at an intensity of $50-85 \%$ of maximum heart rate reserve (\% HRR) or $50-85 \%$ $\dot{V}_{\mathrm{O}_{2} \text { max }}$ with a frequency of 3-5 days week ${ }^{-1}$. However, there is no specific mention of aerobic dance/indoor fitness sessions in the ACSM guidelines. The intensity of effort of the participants in the popmobility sessions has been monitored using a Sport Tester PE3000 heart rate monitor (Polar Electro, Kempele, Finland). However, a recent aerobic dance study conducted by Parker et al. ${ }^{6}$ found that the equivalent exercise oxygen consumption to that during aerobic dance while running on a treadmill resulted in a $10 \%$ higher heart rate in the aerobic dance session. Therefore, Parker et al. ${ }^{6}$ found that the heart rate response during an aerobic dance session overestimated the exercise intensity.

It is not possible to provide the large number of participants in the popmobility sessions with PE3000 heart rate recorders. Participants are asked to monitor heart rate by palpation of the radial or carotid artery. However, this technique is often subject to considerable error and may result in subjects exercising at an inappropriate intensity. The substitution of heart rate monitoring with a rate of perceived exertion (RPE) 15-point scale ${ }^{7}$ could have advantages if it could be shown that RPE scores were closely related to oxygen uptake. Birk and Birk ${ }^{8}$ have previously claimed that the Borg ratings of perceived exertion chart scores of 12-15 exhibit strong correlations with 58-89\% $\dot{V}_{2 \text { max }}$. If a similar relationship held true for RPE scales and oxygen consumption during a popmobility class, the use of RPE scales in the exercise prescription could be of value.

Many of the participants in popmobility sessions wish to maintain their current body weight or decrease their body weight. The $\mathrm{ACSM}^{5}$ have also suggested that a weight-loss programme should provide a negative energy balance not greater than $500-1000 \mathrm{kcal} \mathrm{day}^{-1}$ lower than recommended (and 
not less than $1200 \mathrm{kcal} \mathrm{day}^{-1}$ for adults). A combination of reduced energy intake and increased energy expenditure is suggested for optimal results. According to ACSM energy expenditure should be $300-500 \mathrm{kcal}$ per exercise session. Thus an investigation of the energy cost of the popmobility sessions would provide information on the potential for fat loss if subjects participated in these sessions regularly.

The aims of this study were to measure: the intensity of a popmobility session as determined by oxygen consumption and heart rate; RPE during a popmobility session and compare the RPE with oxygen consumption; the energy cost of a popmobility session.

\section{Methods}

Ten women students of mean(s.d.) age 21.2(1.5) years, volunteered for the study. All subjects had attended popmobility sessions three to five times per week for at least 5 months before the study. Regular exercisers were chosen because they would be familiar with the popmobility movements. The study was approved by the local Ethical Committee before commencing and all subjects completed a consent form and a medical activity history questionnaire before their first test.

Four tests were performed by each subject. A maximum oxygen uptake $\left(\dot{V}_{\mathrm{O}_{2} \text { max }}\right)$ test followed by a popmobility session in a laboratory and a popmobility session in the gymnasium were carried out on separate days.

\section{Test 1 - maximal oxygen uptake test}

Maximal oxygen uptake was measured by a continuous treadmill test. An example of a maximum protocol is given in Table 1.

Over the first $5 \mathrm{~min}$ the treadmill speed was increased. Thereafter, the speed was held constant and a $2 \%$ increase in gradient took place every minute. This procedure was adopted so that the subjects were exhausted around of $10 \pm 2$ mins $^{9}$. The subject wore a noseclip and mouthpiece which was attached to a Hans Rudolph 2600 valve (Cranlea,

Table 1. Example of maximum treadmill protocol

\begin{tabular}{lcc}
\hline Time $(\min )$ & Grade $(\%)^{*}$ & Speed (m.p.h.)t \\
\hline $0-1$ & 0 & 3.0 \\
$1-2$ & 0 & 4.2 \\
$2-3$ & 0 & 4.5 \\
$3-4$ & 0 & 5.0 \\
$4-5$ & 0 & 5.5 \\
$5-6 \ddagger$ & 2 & 5.5 \\
$6-7 \S$ & 4 & 5.5 \\
$7-8$ & 6 & 5.5 \\
$8-9$ & 8 & 5.5 \\
$9-10$ & 10 & 5.5 \\
$10-11$ & 12 & 5.5 \\
$11-12$ & 14 & 5.5 \\
\hline
\end{tabular}

*Increments in gradient always as shown; +Variable speed depending on subjects heart rate response during warm-up run; ¥Noseclips and mouthpiece on at 5.30; §Gas samples taken every minute until the end of the test
Birmingham, UK) which was connected to the Douglas Bag (Cranlea, Birmingham, UK) by a length of tubing. Expired air was analysed immediately after each test using a Taylor Servomex 570A oxygen analyser (Servomex, Crowborough, UK) and a P.K. Morgan 801A carbon dioxide analyser (Morgan, Rainham, UK). Both analysers were calibrated before each test. A Harvard volume meter (Cranlea, Birmingham, UK) was used to measure volume.

\section{Test 2 - popmobility session in the laboratory}

A popmobility session was conducted in a laboratory. Subjects performed this test individually. Heart rate was recorded every $15 \mathrm{~s}$ throughout the $30-\mathrm{min}$ session using the memory mode of a PE3000 Sport Tester (Polar Electric, Kempele, Finland). Expired gas was collected continuously in Douglas Bags (1002001 size) which were held by an experimenter in front of the subject. The Douglas Bags were connected to the subject by a $1.5-\mathrm{m}$ length of flexible tubing attached to the mouthpiece which was connected to a Hans Rudolph 2600 valve. Subjects wore a noseclip. The session was performed to a self-instruction tape which gave exercise commands in time to the music (without visual aid). All subjects had previously practised the session at least twice in a group session to ensure movement terminology would be understood. The $30-\mathrm{min}$ session and corresponding gas collection times were as follows:

Part 1: Aerobic section

6 bags for $3 \mathrm{~min}, 3,3,4,4,3$ - total $20 \mathrm{~min}$.

Part 2: Local muscular endurance section

2 bags for $2,3 \mathrm{~min}$ - total $5 \mathrm{~min}$.

Part 3: Flexibility section

$1 \mathrm{bag}$ for $5 \mathrm{~min}$ - total $30 \mathrm{~min}$.

During bag changeovers the tubing was open to the atmosphere for no more than $2 \mathrm{~s}$ per changeover resulting in a negligible loss of gas (less than $1 \%$ of the total time). Subjects gave a rating on the 15-point Borg RPE scale ${ }^{7}$ every $5 \mathrm{~min}$. Gas analysis was conducted as for test 1. Energy expenditure was calculated using the Weir formula ${ }^{10}$.

\section{Test 3 - popmobility session in the gymnasium}

A popmobility session was performed in a gymnasium within a normal group popmobility session. The subjects performed the same movements to the same music as test 2 while wearing a PE3000 Sport Tester.

During tests 2 and 3, subjects were instructed to work at their normal pace.

\section{Test 4 - percentage fat, body mass and height}

Percentage body fat was estimated using the skinfold method outlined by Durnin and Womersley ${ }^{11}$. Subjects were weighed, and skinfold was measurement carried out and height taken before the $\dot{V}_{\mathrm{O}_{2} \max }$ test. 
Training zones $(50-85 \%)$ for each subject were determined for $\dot{V}_{O_{2 \max }}$ and \% HRR. Resting heart rates were recorded by subjects in bed before rising to enable $\%$ HRR to be calculated ${ }^{12}$.

\section{Results}

The physical characteristics of the subjects are given in Table 2.

The maximum values for the maximum uptake test are given in Table 3. A comparison of mean heart rate between laboratory and gymnasium heart rates is shown in Figure 1. An analysis of variance with repeated measures showed that there were no differences in the first $20 \mathrm{~min}$ of the sessions. During the last $10 \mathrm{~min}$ there was a non-systematic difference in heart rate between the laboratory and gymnasium sessions.

The mean percentage $\dot{V}_{\mathrm{O}_{2} \text { max }}$ and standard deviations for the 30-min session (subdivided into bag collection times) are given in Figure 2. Only one subject was below $50 \% \dot{V}_{\mathrm{O}_{2} \text { max }}$ at any time during the aerobic section $(48 \%$ in the first bag). All but one subject (at bag 7) showed a decreased intensity to

Table 2. Physical characteristics of subjects

\begin{tabular}{lcc}
\hline Characteristics & Mean(s.d.) & Range \\
\hline Height (m) & $1.657(0.04)$ & $1.575-1.695$ \\
Weight $(\mathrm{kg})$ & $58.6(4.3)$ & $50.8-64.6$ \\
Fat (\%) & $23.6(3.8)$ & $16.8-29.1$ \\
\hline
\end{tabular}

Table 3. Maximum values attained in treadmill test

\begin{tabular}{lc}
\hline Parameters tested & Mean(s.d.) \\
\hline$\dot{V} \mathrm{O}_{2}\left(\mathrm{I} \mathrm{min}^{-1}\right)$ & $2.67(0.228)$ \\
$\dot{\mathrm{O}_{2}}\left(\mathrm{ml} \mathrm{kg}^{-1} \min ^{-1}\right)$ & $45.67(4.16)$ \\
$\mathrm{Heart}_{\text {rate max }\left(\text { beats } \min ^{-1}\right)}$ & $203.7(0.05)$ \\
Respiratory exchange ratio & $1.17(0.05)$ \\
Treadmill time & $10 \mathrm{~min} 30 \mathrm{~s}(53 \mathrm{~s})$ \\
\hline
\end{tabular}

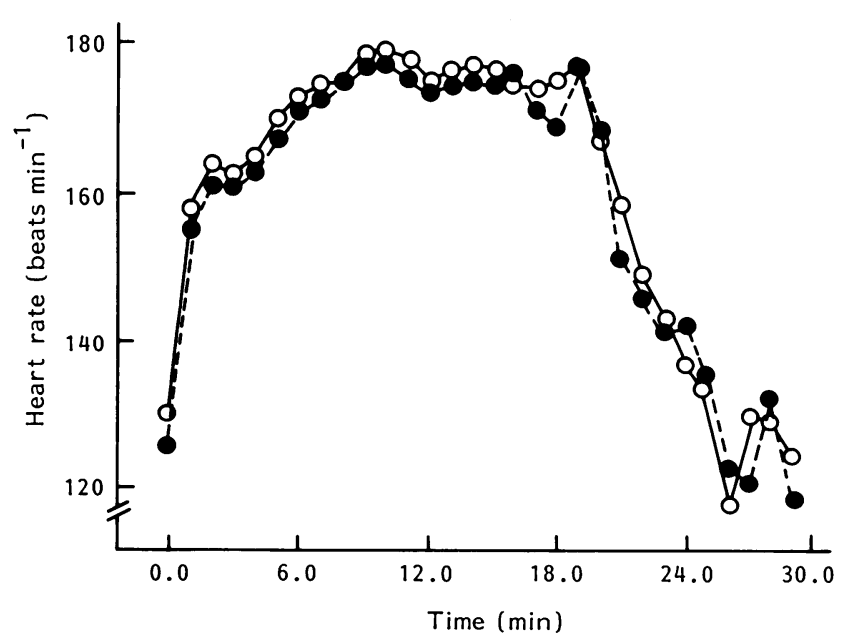

Figure 1. A comparison of mean heart rates between laboratory and gymnasium popmobility sessions. $O$, gymnasium session; , laboratory session below $50 \% \dot{V}_{\mathrm{O}_{2}}$ in the last $10 \mathrm{~min}$ of the session. The mean for the aerobic section was $76.4 \% \dot{V}_{\mathrm{O}_{2} \max }$.

The mean percentage heart rate reserve and standard deviations for the 30-min session (subdivided into bag collection times) are given in Figure 3. All subjects were above $50 \%$ of maximum heart rate reserve during the aerobic section and the first $2 \mathrm{~min}$ of the muscle conditioning. The mean for the aerobic section was $75.6 \%$ HRR.

Rate of perceived exertion scores are shown in Figure 4 with the mean percentage $\dot{V}_{\mathrm{O}_{2}}$ max at the time

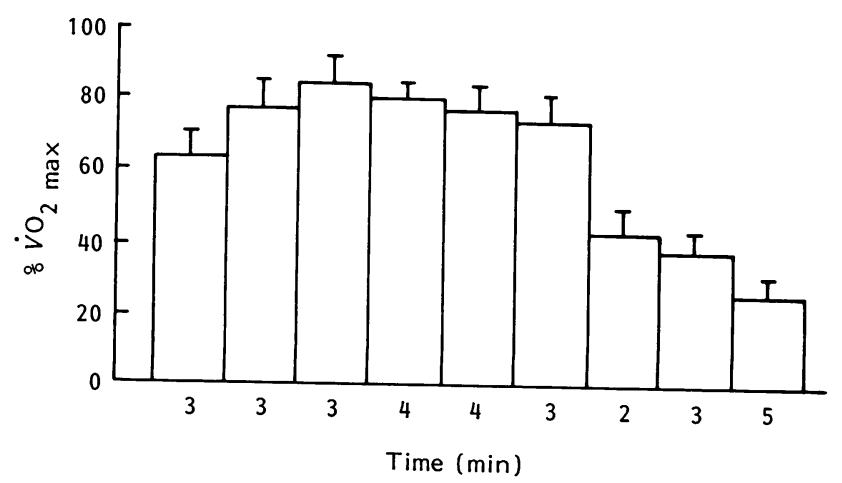

Figure 2. Mean $\% \dot{V}_{\mathrm{O}_{2} \max }$ versus time. The time relates to the collection time for each gas sample

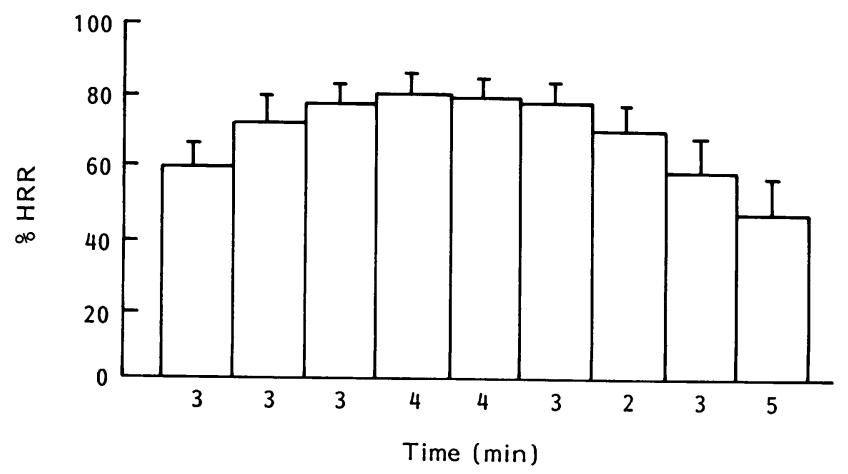

Figure 3. Mean(s.d.) \% HRR versus time. The time relates to the collection time for each gas sample

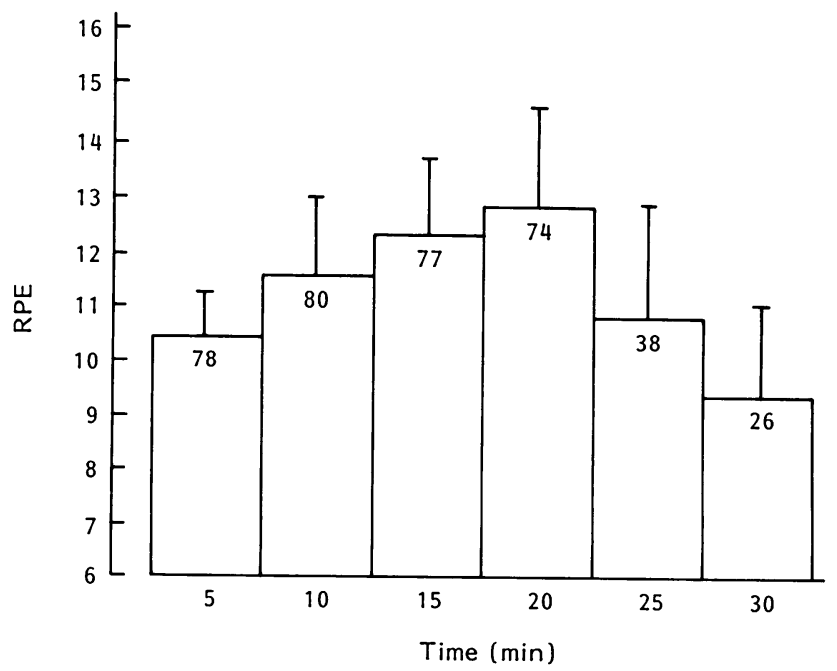

Figure 4. The rate of perceived exertion taken at the end of each 5-min period and the mean(s.d.) $\% \dot{V}_{O_{2} \max }$ at that time 


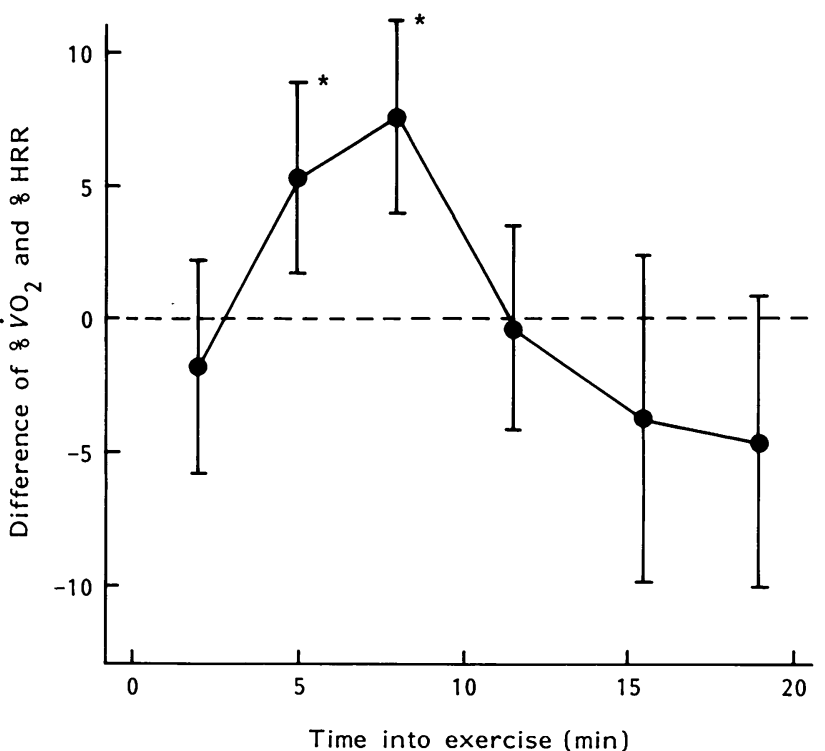

Figure 5. The difference of $\% \dot{V}_{\mathrm{O}_{2}}$ and $\%$ HRR. ${ }^{*} 95 \%$ confidence intervals show that there are significant differences between $\% \dot{V}_{\mathrm{O}_{2}}$ and \% heart rate at these times (i.e. $P$ values $<0.05$ )

of the assessment of the RPE scores. RPE scores ranged from six (the lowest on the chart) during the muscle conditioning and flexibility sections to 15 during the aerobic section. RPE scores increased despite a very similar $\dot{V}_{2}$ throughout the aerobic session.

An analysis of variance with repeated measures was used to investigate the differences in $\% \dot{V}_{\mathrm{O}_{2} \text { max }}$ and $\%$ HRR. Figure 5 demonstrates that the difference of $\% \dot{V} \mathrm{O}_{2 \max }$ and \% $\mathrm{HRR}$ is significantly different from zero between 3 and $9 \mathrm{~min}$.

Table 4 gives the individual and mean energy expenditure of the 10 subjects for the laboratory popmobility session.

The mean(s.d.) total energy expenditure was 236.6(28.4) kcal (range 203-288).

\section{Discussion}

The ACSM (1990) guidelines indicated that intensity should be between $50-85 \% \quad \dot{V}_{\mathrm{O}_{2} \max }$ and maximum

Table 4. Individual, mean energy expenditure and standard deviation for all ten subjects for the laboratory popmobility session

\section{Subject number}

Energy expenditure ( $k c a l)$

\begin{tabular}{rr}
\hline 1 & 287.7 \\
2 & 263.2 \\
3 & 219.0 \\
5 & 216.1 \\
6 & 235.1 \\
7 & 212.1 \\
8 & 261.8 \\
9 & 202.7 \\
\hline
\end{tabular}

Mean(s.d.) $=236.6(28.4) \mathrm{kcal}$ heart rate reserve (HRR) to improve aerobic fitness. The mean $\% \quad \dot{V}_{\mathrm{O}_{2} \max }$ of $76.4 \%$ and mean HRR of $75.6 \%$ for the aerobic section clearly show that the intensity as determined by oxygen consumption and heart rate is high enough to elicit a training effect. Aerobic dance studies have shown $\dot{V}_{\mathrm{O}_{2} \text { max }}$ intensities of $80.3 \%{ }^{12}, 78.14 \%{ }^{13}$ and $70 \%{ }^{14}$. Thus it seems that the mean value of $76.4 \%$ from this study compares favourably with similar studies. Indeed, similar improvements in $\dot{V}_{\mathrm{O}_{2} \max }$ to aerobic training studies could be expected if popmobility sessions were performed three to five times per week.

Davis and Convertino ${ }^{15}$ indicated that the heart rate reserve and $\dot{V}_{O_{2 m a x}}$ are interchangeable. This study shows that monitoring of heart rate would seem to be an appropriate indicator of intensity in a popmobility session when \% HRR is compared with $\% \dot{V} \mathrm{O}_{2 \text { max. }}$

While the mean \% $\dot{V}_{2}$ max and mean \% HRR in this study are fairly closely matched, these findings contrast markedly with Parker et al. ${ }^{6}$ who found a large difference between the \% $\dot{V}_{\mathrm{O}_{2} \max }$ and the $\%$ HRR. Parker et al. ${ }^{6}$ observed that the $86.8 \%$ HRR was much higher than the 'oxygen intensity' of $62.3 \%$. The heart rates were compared to the heart rate response during treadmill jogging at the same $\dot{V}_{2}$ as the dance session. The heart rate proved to be significantly lower at the same $\dot{V}_{2}$ as the dance session. The authors postulate that the elevation in heart rate during aerobic dance is due to a greater amount of arm movement (especially at or above shoulder height) which, according to Astrand et al. ${ }^{16}$, causes an increase in sympathetic tone which would increase heart rate. The findings of Williford et al. ${ }^{13}$ are more in line with the popmobility results. Williford et al. ${ }^{13}$ found heart rate responses of $83.6 \%$ HRR max and $\dot{V}_{\mathrm{O}_{2}}$ values of $78.1 \% \mathrm{VO}_{2 \max }$ for a high impact routine.

When heart rates are used to estimate percentage $\dot{V}_{\mathrm{O}_{2 m a x}}$ this method presupposes that there is a linear relationship between heart rate and oxygen consumption. An added complication is the time effect on the heart rate $/ \dot{V}_{2}$ relationship. In the period from 3 to $9 \mathrm{~min}$ the difference of $\% \dot{V O}_{2}$ and \% HRR was significantly different from zero. However, at most the magnitude of difference was $7 \%$ which is equivalent to around nine beats $\min ^{-1}$. Thus, heart rate monitoring in a popmobility session is a fairly accurate indication of intensity.

A further study is needed to compare the heart rate/ $\dot{V}_{2}$ relationship between a popmobility session and treadmill running. Furthermore, the maximum heart rate may be lower in a popmobility session.

The similar exercise heart rates in the gymnasium and laboratory during the first 20 min suggest that the gas collection equipment did not inhibit participation in the laboratory. Thus, it is believed that the laboratory session was representative of the activity carried out in the gymnasium. The reason for the significant difference in heart rates in the last $10 \mathrm{~min}$ between the gymnasium and laboratory sessions is unknown.

RPE values were not found to be accurate indicators of the measured aerobic intensity according to Birk and Birk $^{8}$ guidelines. While the relative 
oxygen consumption remained fairly static for the aerobic section, the RPE score rose. The RPE values were low compared to the scores which Birk and Birk $^{8}$ suggest for the relative intensity of $75 \% \dot{V}_{\mathrm{O}_{2} \text { max }}$. Possible explanations of this could be that the participants may not have realized how hard they were working because they were engrossed in the session and concentration on the movements along with loud pop music may have distracted the participants sufficiently that they were unaware of the true intensity. Fatigue factors could be implicated in the progressive increase in RPE throughout the aerobic session despite a relatively stable $\dot{V}_{\mathrm{O}_{2}}$. Therefore RPE as a measure of intensity would appear to be unsuitable for exercise prescription for popmobility sessions.

The mean energy expenditure of $237 \mathrm{kcal}$ per session does not trigger the minimum energy expenditure of $300 \mathrm{kcal}$ per session recommended by $\mathrm{ACSM}^{5}$. However, the $\mathrm{ACSM}^{5}$ guidelines acknowledge the suggestion of Haskell ${ }^{17}$ and Haskell et al. ${ }^{18}$, that energy expenditure calculations should consider the subject's body weight. Haskell suggests an energy expenditure of $4 \mathrm{kcal} \mathrm{kg}^{-1}$ of body weight per person. Thus, the mean body weight of $58 \mathrm{~kg}$ in this study would result in a minimum energy expenditure threshold of $232 \mathrm{kcal}$ per session. Regular participation in popmobility sessions could result in a decrease in body fat.

The Glasgow University popmobility session is of adequate intensity to improve aerobic fitness of its participants. Heart rate used as a measure of intensity during a popmobility session would appear to be a fairly accurate indicator of intensity. However, the use of RPE for exercise prescription in popmobility sessions is inappropriate. Popmobility could also be useful in a weight-reduction programme.

\section{References}

1 Dowdy DB, Cureton KJ, DuVal HP, Outzs HG. Effects of aerobic dance on physical work capacity, cardiovascular function and body composition of middle-aged women. Res Quart 1985; 56: 227-33.

2 Johnson S, Berg K, Latin R. The effect of training frequency of aerobic dance on oxygen uptake, body composition and personality. J Sports Med Phys Fitness 1984; 24: 290-8.

3 Vaccaro $\mathrm{P}$, Clinton $\mathrm{M}$. The effects of aerobic conditioning on the body composition and maximal oxygen uptake of college women. J Sports Med Phys Fitness 1981; 21: 291-4.

4 Clearly M, Moffat R, Knuteen K. The effects of two and three day per week aerobic dance programme on maximal oxygen uptake. Res Quart 1984; 55: 172-4.

5 American College of Sports Medicine. The recommended quantity and quality of exercise for developing and maintaining cardiorespiratory and muscular fitness in healthy adults. Med Sci Sports Exerc 1990; 22: 265-74.

6 Parker SB, Hurley BF, Hanlon DP, Vaccaro P. Failure of target heart rate to accurately monitor intensity during aerobic dance. Med Sci Sports Exerc 1989; 21: 291-4.

7 Borg GAV. Psychophysical bases of perceived exertion. Med Sci Sports Exerc 1982; 14: 377-81.

8 Birk TJ, Birk CA. Use of ratings of perceived exertion for exercise prescription. Sports Med 1987; 4: 1-8.

9 Buchfuhrer MJ, Hansen JE, Robinson TE, Sue DY, Wasserman $\mathrm{K}$, Whipp BJ. Optimizing the exercise protocol for cardiopulmonary assessment. J Appl Physiol 1983; 55: 1558-64.

10 Weir JB de V. New methods for calculating metabolic rate with special reference to protein metabolism. J Physiol 1949; 109: 1-9.

11 Durnin JVGA, Womersley J. Body fat assessed from total body density and its estimation from skinfold thickness measurements on 481 men and women aged from 16-72 years. Br J Nutr 1974; 32: 77-97.

12 Claremount AD, Simouitz SA, Boarman MA, Asbell AO, Auferoth SJ. The ability of instructors to organise aerobic dance exercise and effective cardiovascular training. Physician and Sportsmedicine 1986; 14(10): 89-101.

13 Williford HN, Olsen MS, Blessing DL. The physiological effects of aerobic dance - a review. Sports Med 1989; 8: 335-45.

14 Igbanugo V, Gutn B. The energy cost of aerobic dancing. Res Quart 1978; 49: 308-16.

15 Davis JA, Convertino VA. A comparison of heart rate methods for predicting endurance training intensity. Med Sci Sports Exerc 1975; 7: 295-8.

16 Astrand I, Guharay A, Wahren J. Circulatory responses to arm exercise with different arm positions. J Appl Physiol 1968; 25: 528-32.

17 Haskell WI. Physical activity and health: need to define the required stimulus. Am J Cardiol 1985; 55: 40-90.

18 Haskell WI, Montoyne HJ, Orinsinn D. Physical activity and exercise to achieve health related physical fitness components. Public Health Report 1985; 100: 202-12. 